\title{
A Technical Modification to Reduce Potential Contamination in Transperineal Prostate Fusion Biopsy
}

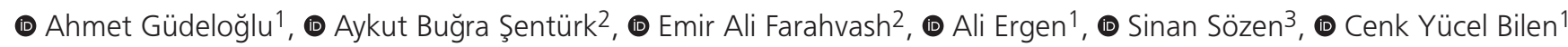 \\ 1 Hacettepe University Faculty of Medicine, Department of Urology, Ankara, Turkey \\ 2 Lösante Children's and Adult Hospital, Clinic of Urology, Ankara, Turkey \\ 3 Gazi University Faculty of Medicine, Department of Urology, Ankara, Turkey
}

\begin{abstract}
Objective: Prostate fusion biopsy could be performed either transrectal or transperineal route. Interestingly, the risk of infective complications after transperineal approach does not seem fewer than transrectal approach. Even when transperineal approach is chosen surgeons may have to deal with fecal contents during the biopsy procedure. We have developed a simple modification to minimize perineal contamination during transperineal prostate fusion biopsy. In this study, the results of patients who underwent transperineal fusion biopsy by using our novel technique were presented.

Materials and Methods: Targeted prostate fusion biopsy was performed in 91 patients who underwent mutliparametric magnetic resonance imaging (Philips Intera $3.0 \mathrm{~T}$ ) and had Prostate Imaging Reporting and Data System 3 or higher lesions, under general anesthesia, with the guide of transrectal ultrasonography (SmartUS, Telemed), with the help of transperineal stepper (CIVCO, EX3) by using MIM Symphony (MIM Software, Cleveland) software between May 2016 and May 2019 with a suspicion of prostate cancer. In the first 24 patients, both targeted fusion biopsy and systematic biopsy were taken only by transperineal approach, while in the 67 patients who were followed up, fusion biopsy was taken by transperineal method and systematic biopsy was taken by transrectal method. In the original technique, a condom filled with $120 \mathrm{~mL}$ saline was placed into the rectum; whereas in our modified technique, initially an empty condom was placed into the rectum and filled with $100 \mathrm{~mL}$ ultrasound gel and then fixed to the anus by using a standard ileostomy adapter.

Results: We performed only transperineal biopsy in 24 patients and both transperineal and transrectal biopsies in 67 patients. A total of 139 lesions with an average size of 9.6-4.4 mm (4-25) were sampled by transperineal route in 91 patients. The mean number of core biopsies was 5.2-1.7 (2-13) for each of 139 lesions. Pathological examination revealed prostate adenocarcinoma in 33 lesions (23.7\%) out of 139 lesions. None of the patients had fever or urosepsis after transperineal approach however, only $1(p=0.43)$ patient who underwent transperineal and transrectal biopsies developed urosepsis symptoms and treated with intravenous antibiotic in the hospital.

Conclusion: Our novel and simple technique to reduce infective complications after transperineal biopsies seems feasible and safe. Further comparative studies with larger number of patients are needed to demonstrate its clinical benefit.
\end{abstract}

Keywords: Prostate biopsy, fusion biopsy, urosepsis

\section{Introduction}

Combining imaging modalities such as mutliparametric magnetic resonance imaging $(\mathrm{mpMRI})$ and transrectal ulrasonography (TRUSG) has entered clinical practice as an exciting innovation, potentially increasing the diagnostic capacity of TRUSG-guided standard biopsy.

mpMRI/USG fusion biopsy can be applied transrectal or transperineal, depending on the software platform used. In transrectal practice, prostatitis and urosepsis are observed in approximately $5 \%$ due to the unavoidable flora of the rectum and rectal inoculation, and it has been reported that this rate has increased in recent studies due to increased fluoroquinolone resistance (1). Interestingly, it has been shown that although there are no bacteria in the perineum as much as the rectum and there is no transrectal inoculation, there is no difference between transperineal and transrectal approach in terms of prostatitis and urosepsis rates $(2,3)$. One potential explanation

Cite this article as: Güldeoğlu $A$, Şentürk $A B$, Farahvash EA, Ergen A, Sözen S, Bilen CY. A Technical Modification to Reduce Potential Contamination in Transperineal Prostate Fusion Biopsy. Bull Urooncol 2020;19(2):60-63 
may be that the perineum is contaminated with a transrectal ultrasound probe that is moved through the rectum during the procedure.

In this study, a simple modification technique to reduce perineal contamination during transperineal prostate fusion biopsy was demonstrated and it was aimed to present the results of the patients to whom this technique was applied.

\section{Materials and Methods}

Targeted prostate fusion biopsy was performed in 91 patients who underwent mpMRI (Philips İntera 3.0 T) and had Prostate Imaging Reporting and Data System (PI-RADS) 3 or higher lesions, under general anesthesia, with the guide of transrectal USG (SmartUS, Telemed), with the help of transperineal stepper (CIVCO, EX ${ }^{3}$ ) by using MIM Symphony (MIM Software, Cleveland) software between May 2016 and May 2019 with a suspicion of prostate cancer. In the first 24 patients, both targeted fusion biopsy and systematic biopsy were taken only by transperineal approach, while in the 67 patients who were followed up, fusion biopsy was taken by transperineal method and systematic biopsy was taken by transrectal method.

In the standard transperineal biopsy approach performed in lithotomy position under general anesthesia, the condom filled with $100-120 \mathrm{~mL}$ of saline is attached to the transrectal ultrasound probe and the probe is placed in the rectum in connection with this condom. During this procedure, the perineum is exposed to continuous rectal contamination by the manipulation of the probe. In the technical modification we have developed, $50 \mathrm{~mL}$ ultrasound gel is injected into the rectum at the lithotomy position under general anesthesia with the help of a irrigation syringe. Then the empty condom is placed in the rectum and filled with $100 \mathrm{~mL}$ ultrasound gel and then the condom is mounted on the ileostomy adapter, the portion over the perineal region is tangentially cut off so that the adapter is fixed to the anal region. The perineal area is cleaned with batticon and then covered with sterile off-site. Only the portion of the off-site inside the ileostomy adapter is cut, thus providing a completely sterile surgical area (Figure 1). After this stage, the transrectal ultrasound probe moves comfortably back and forth in the rectum, preventing the perineum from being re-contaminated with the rectal content.

After this procedure under general anesthesia, all patients were discharged on the same day.

\section{Statistical Analysis}

Statistical analysis in this study was made using the SPSS v.20 (IBM Corp.) package program. The chi-squared test was used to compare the relationship between variables with two categories.

\section{Results}

Of the total 91 patients, 24 only underwent transperineal biopsy and 67 underwent both transperineal fusion biopsy and transrectal systematic biopsy. In 15 of the 24 patients who underwent only transperineal biopsy, both transperineal fusion biopsy and transperineal systematic sampling were performed. In 9 of the 24 patients, only a targeted transperineal fusion biopsy was performed. The mean prostate volume was $55 \pm 21.6$ $\mathrm{cm}^{3}$ (20-120), the mean age was $61 \pm 7.9$ years $(42-85)$ and the mean prostate specific antigen (PSA) value was $7.7 \pm 8.2 \mathrm{ng} /$ $\mathrm{mL}$ (1.15-64). In 14 patients (15\%), digital rectal examination was positive. Eighteen patients (20\%) had a history of standard

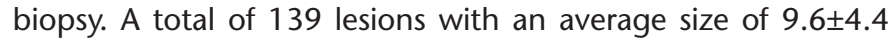
$\mathrm{mm}$ (4-25) were sampled in 91 patients. The percentages of the lesions located in the apex, mid, base, anterior, TZ, apex-mid and base-mid regions were 18.7\%, 36\%, 18.7\%, 1.4\%, 9.4\%, $8.6 \%$ and $7.2 \%$, respectively. Of the lesions, $51.8 \%(72 / 139)$ were classified as PI-RADS 3, 36\% (50/139) as PI-RADS 4, and $12.2 \%$ (17/139) as PI-RADS 5. An average of 5.2 \pm 1.7 (2-13) core biopsies was taken from each lesion by transperineal route. As a result of pathological examination, prostate adenocarcinoma was detected in 33 (23.7\%) of 139 lesions. The mean duration of anesthesia for patients was 60 minutes (45-100).

While no post-op urosepsis was observed in 24 patients who underwent only transperineal biopsy, only $1(p=0.43)$ patient who underwent both transperineal and transrectal systematic biopsies developed signs of urosepsis within 48 hours of postop period and the patient was hospitalized and treated with intravenous antibiotics. In addition, 1 patient also developed hematuria and urinary retention due to the process.

Through our technical modification, the contamination of the perineum with rectal content has been completely eliminated while at the same time the transrectal ultrasound image quality has been subjectively improved.

\section{Discussion}

Prostate cancer is the most common cancer among men and the second most common cause of death from cancer (4). Transrectal ultrasound assisted biopsy is still the most common method in the diagnosis of prostate cancer. Although transrectal ultrasound is useful for showing specific anatomical regions of the prostate or targeting identifiable lesions, it alone is insufficient to detect localized cancer within the prostate (5). Recent studies have shown that mpMRI obtained before biopsy increases the rate of detection of clinically important prostate cancer and limits the detection of innocent disease (6). Now, in many centres in our country, mpMRI/USG fusion biopsy is used when there is suspicion of clinical prostate cancer, even in the first biopsy. mpMRI/USG fusion biopsy can be done cognitively as well as with software. In the fusion biopsy technique performed using computer software, 2 different approaches are applied, depending on the software being transrectal or transperineal.

In the Global Prevalence study which investigated the infection rate after transrectal prostate biopsy in 702 men from 84 centers around the world showed that symptomatic urinary tract infection (3.5\% had fever, 3.1\% required hospitalization) was observed in $5.2 \%$ of the patients, despite $90 \%$ of patients were given prophylactic fluoroquinolone (7). In our country, Şimşir et al. (8) examined urosepsis rates after more than 2000 transrectal biopsies performed in their centers and showed that $3.06 \%$ of patients developed urosepsis within the first 5 days after the procedure. After 72500 TRUSG assisted biopsies performed in the UK in $2008,2.5 \%$ to $3.6 \%$ of patients were 

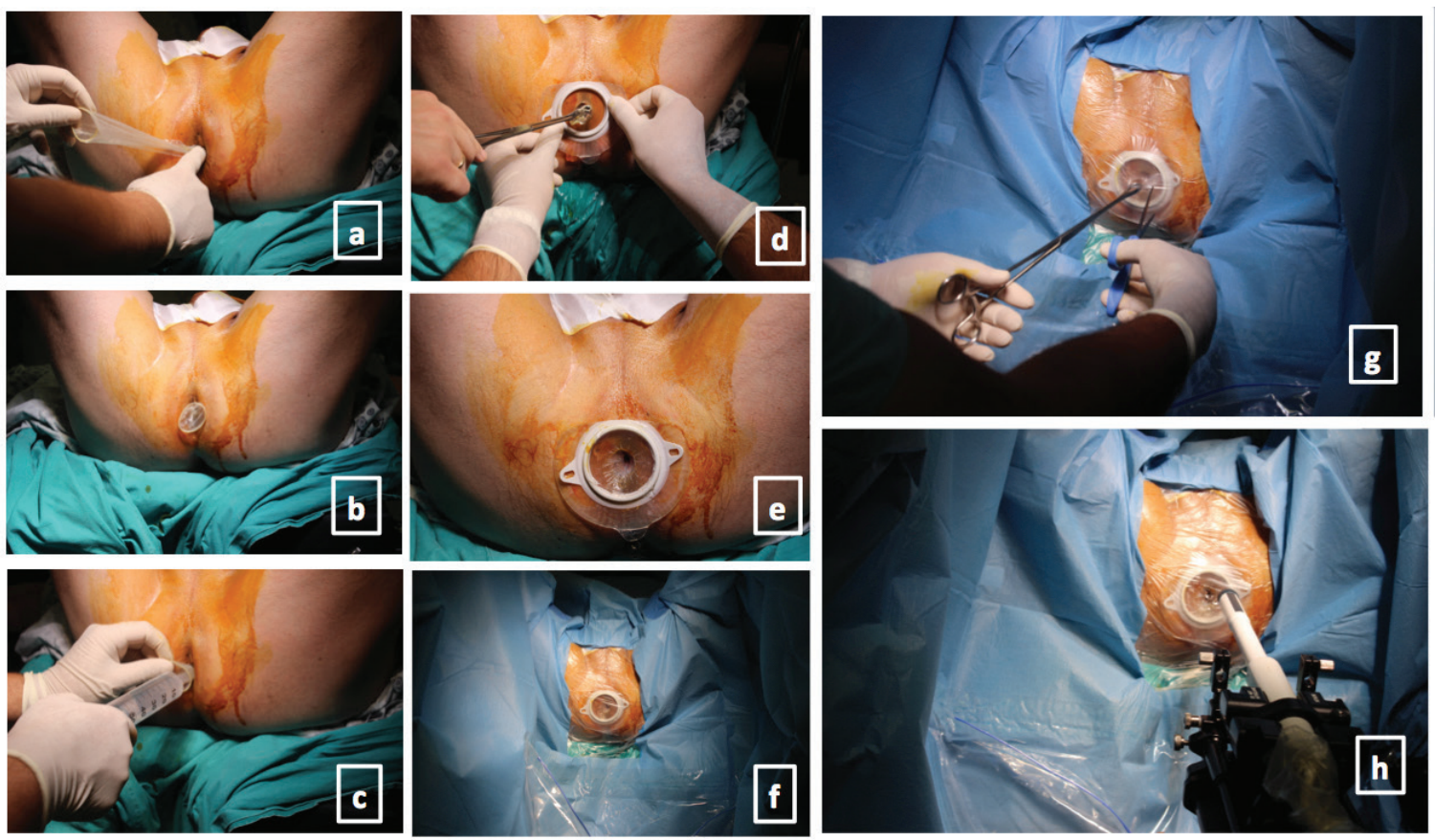

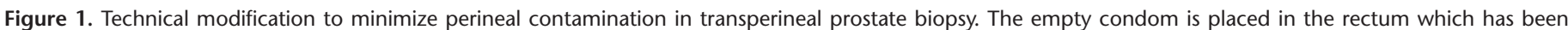

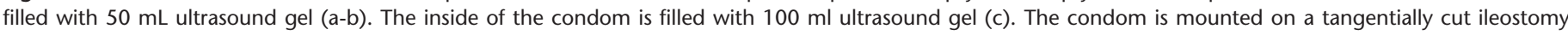

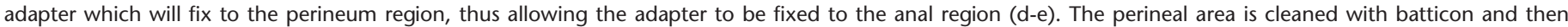
covered with sterile off-site (f). Only the part of the off-site that comes into the ileostomy adapter is cut off, thus providing a completely sterile surgical area (g-h).

re-admitted in the hospital due to the process-related infection and the annual cost of this complication was estimated to be approximately $£ 1$ million (9). Çam et al. (10) showed that after TRUSG assisted biopsies performed in our country, 2\% of the patients were hospitalized due to infection.

Fluoroquinolone resistance has increased not only in gastrointestinal or urinary tract infections, but in many infectious diseases, such as skin infections or sexually transmitted diseases, and has become a global problem (11). More than $75 \%$ of E.coli, which causes infections after prostate biopsy, have been shown to be quinolone resistant in our country (12). Factors that increase fluoroquinolone resistance can be classified as patientrelated and process-related factors (1). Patient-related factors are co-morbidities such as diabetes or chronic obstructive pulmonary disease, travel to foreign countries, benign prostatic hyperplasia, urogenital infection, history of recent antibiotic use (especially fluoroquinolone), the presence of a urethral catheter, having recently been hospitalized or being a hospital employee and positive culture before biopsy. A large number of biopsies, repeated biopsies and contaminated ultrasound gel are factors related to the process. The presence of a fluoroquinoloneresistant microorganisms in rectal swab culture is also an important predictor of infection development after prostate biopsy (13). In the light of all this information, whether it is transrectal or transperineal, the complication of infection after prostate biopsy is undeniably serious and costly. The fact that fluoroquinolone-resistant microorganisms are growing globally and nationally suggests that this problem will also appear as an important clinical problem in the coming years.
Several strategies have been applied to prevent infective complications after prostate biopsy. First of all, patients should be evaluated individually before the procedure and the risk factors such as accompanying co-morbidity, urologic attempt history, the use of antibiotics in the last 6 months, history of international travel and being a health care worker should be questioned (14). In addition, strategies such as bowel cleansing with povidone-iodine, prophylactic antibiotics before the procedure, or target-oriented antibiotic prophylaxis due to the result of culture from a rectal swab sample have also been developed (14). Other factors such as contamination from multi-use ultrasound gel containers, contamination of the tip of the biopsy needle during transfer of tissue samples to pathological tubes or pads, or contamination via multi-use needle guide should also be considered $(1,14)$.

Another strategy for preventing infection complications due to prostate biopsy is the use of a perineal approach instead of a microorganisms-rich rectal approach. A recently published meta-analysis revealed that there was no difference between the two approaches in terms of complications (3). A potential explanation for this paradox is that despite the pre-operative peraparation of the perineum, it has been re-contaminated with a continuously manipulated ultrasound probe during the procedure placed in the rectum. Supporting this explanation, in our series, 24 patients who underwent only transperineal biopsy had no procedure-related infection as a complication.

\section{Study Limitations}

One of the most important limitations of this study was that it was done with a limited number of patients and that it was a 
retrospective study. In addition, according to our knowledge, the absence of a similar application to reduce the infectious complicatios in the transperineal approach in the literature makes our study unique.

\section{Conclusion}

Infectious complication after prostate biopsy is a major health problem that has the potential to occur more frequently today due to increased fluoroquinolone resistance. Even in the transperineal approach, which is commonly used especially in fusion biopsies, the rate of this complication is not less than the transrectal approach. This simple technical modification, which we have put into clinical practice for the first time, appears to be a new, effective and safe strategy for reducing the complication of infection after transperineal prostate biopsies. Comparative studies with a larger number of patients are needed to demonstrate the clinical benefit of this technical modification.

\section{Acknowledgements}

Publication: The results of the study was published in (Engineerind \& Urological Society Meeting May 12, 2017 Boston/US) and (Focal Therapy \& Imaging in Prostate Cancer \& Kidney Cancer Meeting February 11-13, 2018 Noordwijk/ Netherlands)

Contribution: There is not any contributors who may not be listed as authors.

Conflict of Interest: No conflict of interest was declared by the authors.

Financial Disclosure: The authors declared that this study received no financial support.

\section{Ethics}

Ethics Committee Approval: Retrospective study.

Informed Consent: Retrospective study.

Peer-review: Externally peer-reviewed.

Authorship Contributions

Concept: S.S., C.Y.B., Design: A.E., S.S., C.Y.B., Data Collection or Processing: A.G., Analysis or Interpretation: A.G., S.S., C.Y.B., Literature Search: A.G., S.S., C.Y.B., Writing: A.G., S.S., C.Y.B.

\section{References}

1. Loeb S, Vellekoop A, Ahmed HU, et al. Systematic review of complications of prostate biopsy. European urology 2013;64:876-892.
2. Hara R, Jo Y, Fujii T, et al. Optimal approach for prostate cancer detection as initial biopsy: prospective randomized study comparing transperineal versus transrectal systematic 12-core biopsy. Urology 2008;71:191-195.

3. Shen PF, Zhu YC, Wei WR, et al. The results of transperineal versus transrectal prostate biopsy: a systematic review and meta-analysis. Asian journal of andrology 2012;14:310-315.

4. Siegel RL, Miller KD, Jemal A. Cancer statistics, 2016. CA: A Cancer Journal for Clinicians 2016;66:7-30.

5. Smeenge $M$, Barentsz J, Cosgrove $D$, et al. Role of transrectal ultrasonography (TRUS) in focal therapy of prostate cancer: report from a Consensus Panel. BJU international 2012;110:942-948.

6. Fulgham PF, Rukstalis DB, Turkbey IB, et al. AUA Policy Statement on the Use of Multiparametric Magnetic Resonance Imaging in the Diagnosis, Staging and Management of Prostate Cancer. J Urol 2017; 198:832-838.

7. Wagenlehner FM, van Oostrum E, Tenke $P$, et al. Infective complications after prostate biopsy: outcome of the Global Prevalence Study of Infections in Urology (GPIU) 2010 and 2011, a prospective multinational multicentre prostate biopsy study. European urology 2013;63:521-527.

8. Simsir A, Kismali E, Mammadov R, Gunaydin G, Cal C. Is it possible to predict sepsis, the most serious complication in prostate biopsy? Urol Int 2010;84:395-359.

9. Batura D, Gopal Rao G. The national burden of infections after prostate biopsy in England and Wales: a wake-up call for better prevention. J Antimicrob Chemother 2013;68:247-249.

10. Cam K, Kayikci A, Akman Y, Erol A. Prospective assessment of the efficacy of single dose versus traditional 3-day antimicrobial prophylaxis in 12-core transrectal prostate biopsy. Int J Urol 2008; 15:997-1001.

11. Dalhoff A. Global fluoroquinolone resistance epidemiology and implictions for clinical use. Interdiscip Perspect Infect Dis 2012;2012:976273.

12. Kandemir O, Bozlu M, Efesoy O, Guntekin O, Tek M, Akbay E. The incidence and risk factors of resistant $\mathrm{E}$. coli infections after prostate biopsy under fluoroquinolone prophylaxis: a single-centre experience with 2215 patients. J Chemother 2016;28:284-288.

13. Williamson DA, Masters J, Freeman J, Roberts S. Travel-associated extended-spectrum beta-lactamase-producing Escherichia coli bloodstream infection following transrectal ultrasound-guided prostate biopsy. BJU international 2012;109:E21-2.

14. Liss MA, Ehdaie B, Loeb S, et al. An Update of the American Urological Association White Paper on the Prevention and Treatment of the More Common Complications Related to Prostate Biopsy. J Urol 2017;198:329-334. 